\title{
¿Qué modelo interdisciplinar requiere la neuroética?
}

\section{Which interdisciplinary model does neuroethics require?}

\author{
PAULA CASTELLI
}

Centro de Investigaciones Filosóficas de Buenos Aires. Universidad de Buenos Aires y Universidad

Torcuato Di Tella

Artículo recibido: 2 de julio de 2017

Solicitud de revisión: 19 de septiembre de 2017

Artículo aceptado: 19 de noviembre de 2017

\section{Resumen}

En este trabajo se analizan dos modos paradigmáticos en que en la actualidad se comprende la relación entre las disciplinas que se encuentran implicadas en la tarea de la neuroética, en su carácter de neurociencia de la ética. Particularmente se critica la forma que toma la interdisciplinariedad en estos dos modelos. En el primer caso se señala que la tarea de reflexión filosófica no da lugar a que la evidencia neurocientífica pueda modificar de modo importante los presupuestos teóricos en que se funda tal reflexión. En el segundo caso, por el contrario, el papel dado al componente empírico, necesario a la neuroética, obtura la reflexión ético-filosófica.

Palabras clave: Neuroética, neurociencia de la ética, naturalismo ético y normatividad

\begin{abstract}
In this paper, I analyze two paradigmatic contemporary ways of understanding the relation between the disciplines involved in neuroethics. In particular, I criticize the form interdisciplinarity takes in these two models. It is pointed out that, according to one of these, the philosophical reflection does not allow neuroscientific evidence to influence in any significant way the theoretical presupositions of that reflection. According to the second model, on the contrary, the role of the empirical component, essential to neuroethics, rather blocks the ethical and philosophical reflection.
\end{abstract}

Keywords: Neuroethics, Neuroscience of ethics, Ethical naturalism and Normativity. 
...supongan que uno de ustedes fuera una persona omnisciente y, por consiguiente, conociera los movimientos de todos los cuerpos animados o inanimados del mundo y conociera también los estados mentales de todos los seres que han vivido. Supongan además que este hombre escribiera su saber en un gran libro; tal libro contendría la descripción total del mundo. Lo que quiero decir es que este libro no incluiría nada que pudiéramos llamar juicio ético ni nada que pudiera implicar lógicamente tal juicio.

Conferencia sobre ética

L. Wittgenstein

\section{UN POCO DE HISTORIA}

La neuroética surgió como disciplina hace una década y media. Suele considerarse como evento fundacional la conferencia «Neuroethics: Mapping the Field», impartida en 2002 en San Francisco y, si bien se registran algunos usos del término en escritos anteriores, solo a partir de allí ha tomado el sentido con que lo usamos hoy, es decir, para dar cuenta de un campo disciplinar nuevo (Marcus 2004; Illes 2003; Racine 2010). Aquella conferencia, de la que participaron especialistas de muy diversas disciplinas - neurocientíficos, pero también psicólogos, religiosos, filósofos- se había planteado como meta "proyectar los límites, definir la problemática y plantear las preguntas iniciales apropiadas para un campo que sondea las implicancias éticas del avance en la ciencia del cerebro» (Safire, 2004).

Este sondeo de «implicancias éticas» puede leerse de dos modos distintos y, de hecho, poco después, ese mismo año, aparecía un artículo de Adina Roskies en el que esta autora distinguía dos «ramas» dentro de este nuevo campo: la ética de la neurociencia y la neurociencia de la ética, que se corresponderían respectivamente con una neuroética aplicada y una neuroética teórica (Roskies, 2002). ${ }^{1}$ Si bien esta autora ha reconocido en trabajos posteriores que tal distinción es controvertida y que puede cues-

1 Podemos encontrar autores que establecen otro modo de dar cuenta de los diversos órdenes de investigación en el interior de la neuroética, como es el caso de Northoff (2009), quien distingue entre una neuroética empírica y una neuroética teórica, o Salles y Evers (en prensa) quienes convierten en compleja esta distinción y proponen una distinción tripartita en la que incluyen también una neuroética conceptual. Sin embargo, a los fines de lo que nos interesa discutir en este trabajo, nos parece que la clasificación que establece Adina Roskies resulta más clara. 
tionarse su alcance, ha resultado una distinción sumamente influyente (Roskies, 2016).

La primera de estas ramas no difiere en gran medida de una bioética dedicada específicamente a las cuestiones que surgen como efecto de las nuevas tecnologías que permiten investigar y modificar el cerebro humano de modos en que nunca antes había sido posible. Así, por ejemplo, en el ámbito de la neurología clínica recogería los debates en torno a la muerte cerebral, el estado vegetativo, los estados de mínima conciencia, entre otros. En el ámbito de la investigación sobre el cerebro surgirán cuestiones respecto de la posibilidad de violar la privacidad de los contenidos mentales de los sujetos de investigación, o las posibles aplicaciones de ciertos descubrimientos en áreas como por ejemplo la potenciación cognitiva o la potenciación moral de los individuos. Ciertamente, esta rama de la neuroética implica novedades a nivel disciplinar respecto de otras bioéticas, dada la especificidad del cerebro, pero no es radicalmente distinta de lo que hace la bioética en otros ámbitos, e incluso hay quienes han cuestionado si es necesaria esta delimitación de subdisciplinas dentro de la bioética (Parens \& Johnston, 2007; Evers et al., 2017).

En su segunda acepción, la neuroética consistiría en una neurociencia de la ética.Aquí sí vemos una gran novedad y, tal vez, un campo disciplinar novedoso, pues tiene por objeto el dar cuenta, a partir de una evidencia neurocientífica aportada por las nuevas tecnologías como la resonancia magnética funcional (fMRI), el electroencefalograma cualitativo (qEEG) o la magnetoencefalografía (MEG), del modo en que estos datos sobre el funcionamiento del cerebro humano impactan en nuestro conocimiento acerca de cuestiones morales, sociales y culturales. Particularmente, una rama de la neurociencia, la neurociencia cognitiva, ofrecería explicaciones científicas de relevancia para comprender fenómenos tales como el juicio moral, la agencia moral, la toma de decisiones, la formación del carácter y, en general, la moralidad como fenómeno social (Greene, 2014; Cortina 2011; Joyce 2008).

Mi intención en este trabajo es examinar dos modelos o estilos disciplinares en que se ha desarrollado la neuroética como neurociencia de la ética en los pocos años que tiene de existencia para notar que hay ciertos presupuestos implícitos en estas formas de encarar la cuestión que hacen difícil comprender el papel de la filosofía en el concierto interdisciplinario que supone esta nueva área de conocimiento. Es claro que la neuroética se anuncia, desde su mismo nombre, como un campo de estudio interdisciplinario, en el cual, desde distintos ámbitos, se aporta a un conocimiento 
multifacético y global del fenómeno moral. Mi pregunta aquí es la siguiente: ¿cuál es el modelo metodológico desde el cual debemos pensar la interdisciplinariedad en la neuroética teórica? ¿No hay ciertos modos de pensar esta relación que podrían implicar que la neuroética no es una disciplina filosófica sino más bien una cierta posición dentro del ámbito de la ética? Específicamente una postura naturalista-reductiva sostiene que cuando conozcamos más en detalle el funcionamiento cerebral daremos con la «verdadera teoría ética» y podremos descartar las demás como paradigmas anticuados y erróneos, tal como sostiene Gazzaniga (2005).

Al recorrer los papers y libros en temas de neuroética nos encontramos con estos dos modelos de cooperación que, por otra parte, han comenzado a ser objeto de crítica en los últimos años (Salles \& Evers, en prensa; Giordano, 2011). Ninguno de ellos nos resulta plenamente satisfactorio para pensar la relación entre evidencia neurocientífica y reflexión ética que habría de darse en la neuroética en tanto campo interdisciplinario. Si bien estos dos modelos no son los únicos que encontramos, considero que caracterizan a una parte importante de los trabajos producidos en los últimos años. ${ }^{2}$ Mostraré ambos modelos de interdisciplinariedad, las falencias que veo en ellos y luego trataré de proponer, de un modo muy general, lo que creo que debería ser otro modo de interrelación entre ética y neurociencias que considero más fructífero y que permite pensar que la neuroética pertenece efectivamente al campo de la reflexión filosófica.

\section{EL MODELO DE LA NEUROÉTICA FILOSÓFICA}

Un primer modelo es aquel en el cual la evidencia neurocientífica es usada como soporte de una teoría ética particular, asumida como válida por razones teórico-filosóficas con anterioridad e independencia de los datos empíricos aportados por la neurociencia. En su capítulo «Neuroética y ética del discurso», Adela Cortina (2014) sigue este camino al cuestionarse sobre si es posible encontrar un modelo de neuroética que nos permitirá hacer un tránsito válido del «es cerebral» a un «debe moral» propio de nuestro tiempo. Este «deber» al que se desea articular con el dato empírico neurocientífico lleva implícito ya una serie de conceptos éticos que lo

2 Por ejemplo, Álvarez-Díaz (2013) propone tres modelos de interrelación entre neurociencia y ética a los que nombra como neuroescepticismo, neurorreducionismo y neurocriticismo. Sin embargo, en cuanto a los caracteres que resultan relevantes para nuestro análisis, tanto el neurocriticismo como el neuroescepticismo, con distinto énfasis, son formas en que se plasma el modelo que nosotros llamaremos de la neuroética filosófica. 
fundamentan y a los que, desde esta perspectiva, no se está de ningún modo dispuesto a renunciar, como demuestran las siguientes palabras:

...nos preguntábamos si puede darse el paso deductivo del «es» cerebral al «debe» moral [...] La conclusión es que ese paso no puede darse, entre otras razones, porque del «es» cerebral pueden surgir consejos de prudencia, pero no deberes morales, entendiendo por «moral» el tipo de exigencia que se plantea en el nivel posconvencional en el desarrollo de la conciencia social, que es al que ha accedido nuestro tiempo, como se plasma en declaraciones e instituciones (Cortina, 2014: 188).

Así, por ejemplo, los derechos humanos o la existencia de una dignidad humana son puntos de partida para el análisis filosófico que tienen que ser articulados con los conocimientos que aporta la neurociencia respecto de las bases neurales -que según esta corriente no habría que confundir con fundamentos - de la moralidad humana. En una perspectiva similar, pero partiendo de que la distinción entre hechos y valores no puede ser dejada de lado, Thomas Buller sostiene que «lo que la neurociencia no puede hacer, y no debería estarle permitido hacer, es reemplazar las cuestiones normativas con las científicas» (Buller, 2006: 64).

Desde el punto de vista metodológico, la neuroética así entendida haría uso de las herramientas propias de la filosofía y, en particular en el caso de Cortina, de las de la ética del discurso, mientras que el dato que aporta la ciencia del cerebro cumple el papel de corroborar la validez, tanto de esta metodología como de los conceptos teóricos con los que trabaja, en la medida en que resultan compatibles con esos datos empíricos. En un artículo de 2013, Cortina lo pone en los siguientes términos:

Una neuroética fundamental no puede contentarse con sacar a la luz las bases de la conducta moral, sino que debe preguntarse también por el fundamento de la obligación moral, en la medida en que no puede obviar la pregunta por la validez de los juicios morales. Ciertamente, uno de los temas tradicionales de la ética es el de la fundamentación de la moralidad, y ninguna propuesta filosófica pone en duda que para atribuir una conducta moral a un ser éste debe contar con unas bases corporales, entre las que se encuentra la posesión de un cerebro y un sistema nervioso, articulados con el resto del cuerpo. Pero no es lo mismo «base» que «fundamento», no es lo mismo «condición necesaria» que «Condición suficiente» (Cortina, 2013: 129).

Podría afirmarse, sin embargo, que no hay aquí un conocimiento efectivamente construido de modo interdisciplinario sino, en todo caso, un diálogo entre disciplinas mutuamente ajenas en el que se trata de determinar si están o no hablando sobre lo mismo, si los datos que aporta una de ellas son relevantes y hasta qué punto para las explicaciones teóricas que for- 
mula la otra. ${ }^{3}$ Así, por ejemplo, es importante para este modelo determinar si estas dos disciplinas (neurociencia social y ética) están trabajando sobre un mismo plano, el de dar con las bases neurales de la moralidad o si cada una tiene tareas claramente diferentes en tanto la moralidad no es la ética. En este sentido, esta forma de interdisciplinariedad a la que hemos llamado neuroética filosófica se define por la segunda opción, en la medida en que la ética, como reflexión filosófica sobre el fenómeno moral, no puede sino ser tarea de la filosofía, con independencia de lo que el dato biológico nos aporte respecto de las formas que puede adquirir o ha adquirido evolutivamente la moralidad humana (Conill, 2017; Cortina, 2013).

Creo que es apropiado el nombre de neuroética filosófica para esta tarea, en tanto se trata de una reflexión ético-filosófica que enmarca y da sentido a los descubrimientos sobre el modo en que las conductas humanas descansan en mecanismos neurales. En esta forma de relacionar dato empírico y reflexión ética, las neurociencias son reconocidas como una fuente de información relevante con la que la reflexión filosófica cuenta a la hora de proveer explicaciones teóricas, tal como lo han sido a lo largo de la historia, y lo siguen siendo, muchas otras disciplinas como la psicología, la lingüística, la antropología. Podría hacerse una analogía con el modo en que el adjetivo filosófico es usado para dar cuenta de la antropología filosófica. También allí, la filosofía aporta una serie de conceptos que son relevantes para pensar la cuestión humana, pero no se trata de ningún modo de una empresa interdisciplinaria entre la antropología científica y la filosofía. De igual modo, creo que este modelo de relación entre ética y neurociencia no es interdisciplinario en un sentido fuerte. ${ }^{4}$

\section{EL MODELO DE LA NATURALIZACIÓN}

Un segundo modelo para pensar esta interdisciplinariedad, al que llamaré modelo de la naturalización y que otros, como Álvarez Díaz (2013), llaman neurorreduccionismo, podría describirse del siguiente modo: existen ciertos conceptos con los que ha trabajado tradicionalmente la ética

3 En un sentido semejante, Alfredo Marcos habla de una relación de colaboración entre ética y neurociencia, a lo que llama neuroética en "modo-co" y por la que aboga frente a otro modo de relación entre estas disciplinas en el que la neurociencia sustituiría a la ética filosófica (Marcos, 2015).

4 Por supuesto no estamos negando que haya dentro de esta perspectiva profesionales del ámbito de las neurociencias junto con filósofos colaborando unos con otros. Lo que intentamos analizar es el modelo dentro del cual se da tal colaboración y la concepción de la neuroética que está presupuesta en ella. 
- juicio moral, deliberación, responsabilidad moral o libertad, deber, normatividad-y lo que permite la evidencia neuro, según este modelo, es ver de qué modo el referente de estos conceptos se «encarna» en el funcionamiento cerebral. Si el neurocientífico cognitivista encuentra que no hay anclaje cerebral para cierto concepto, las teorías en las que estos conceptos resulten centrales tendrán que ser descartadas por no tener apoyo en la evidencia empírica (Gazzaniga, 2005).

Joshua Greene podría ser considerado un exponente de esta forma de entender la tarea de la neuroética. En su trabajo «The Secret Joke of Kant's Soul», por tomar solo un ejemplo dentro de su vasta producción en el tema, se propone argumentar, a partir de premisas empíricas, fundadas en evidencia disponible, en las que se afirma que los juicios deontológicos se basan en respuestas emocionales luego racionalizadas. Los juicios morales de corte consecuencialista, en cambio, involucran razonamiento moral genuino, de donde se seguiría que las teorías éticas normativas de carácter deontológico deberían ser puestas en duda (Greene, 2008). Nuestro reproche al argumento de Greene se centra en que realiza con demasiada rapidez y poca prudencia el paso inferencial de lo que puede observarse a partir de imágenes cerebrales (básicamente fMRI) ${ }^{5}$ a la lectura de tales imágenes en clave de conceptos teóricos que describen fenómenos mentales tales como la emocionalidad o el juicio deontológico, para inmediatamente agregar una nueva inferencia que pasa de esta interpretación de la imagen a la afirmación normativa de que deben ser puestas en duda las teorías éticas de corte deontológico.

Desde el punto de vista metodológico, lo que parece estar sucediendo aquí es que, en primera instancia, aun cuando se reconoce que se asumen ciertas posiciones teóricas frente a otras por razones de orden filosófico -por ejemplo, que «deontológico» $\mathbf{y}$ «consecuencialista» son clases naturales psicológicas-, se identifican estos conceptos con ciertos elementos identificables en las imágenes cerebrales como si pudieran correlacionarse uno a uno. Pues no es lo mismo sostener que se iluminan ciertas zonas del cerebro al realizar cierta tarea, que decir que se realiza un juicio deontológico, se activan ciertas emociones o se evalúan consecuencias.

En segunda instancia, se derivan consecuencias normativas a partir de hechos determinables empíricamente, movimiento que exige al menos algún tipo de respuesta a la posible acusación de estar cometiendo la falacia

5 Estas evidencias surgen de las investigaciones que Greene junto con Haidt llevaron a cabo sobre el funcionamiento cerebral en el contexto de resolución de dilemas morales, publicadas en varios artículos a comienzos del milenio (Greene \& Haidt, 2002). 
naturalista. Greene sostiene que el hecho de tener evidencia de que los juicios deontológicos son efecto de intuiciones morales de carácter emotivo, que surgen en nosotros como efecto del particular recorrido evolutivo de la especie humana, invalida toda ética deontológica en tanto tal. Pareciera que en este caso la neurociencia cognitiva despeja a la ética de las ilusiones que la reflexión ética filosófica ha generado. ¿Es necesaria en este modelo la ética como reflexión filosófica? Parece no serlo, dado que el estudio de la naturaleza dará respuesta no solo a cuáles son de hecho los modos humanos de razonamiento moral, sino también a la pregunta por cuál es la justificación correcta de estos razonamientos.

Dentro de este modelo de la neuroética naturalizada, podemos tomar otro caso ejemplar. Patricia Churchland, en su libro El cerebro moral, muestra cómo las hormonas, en particular la oxitocina y la vasopresina, pueden dar una explicación última de las emociones sociales, que compartiríamos con muchos otros mamíferos gregarios. Sobre la base de estas emociones sociales $^{6}$ - pero naturales-, Churchland cree poder explicar de manera completa la moralidad humana. Según ella, de hecho, toda normatividad se reduce a emociones que no pueden fundar sino normas prudenciales que nos permiten satisfacer nuestras necesidades biológicas como especie y que, a lo largo de la historia evolutiva, han tomado formas cada vez más complejas y sofisticadas. Así, por ejemplo, Churchland hipotetiza sobre las razones de la reacción positiva y de apego que se genera en la madre cuando un bebé imita conductas o gestos y sugiere que se origina en que la mímica funciona como un cohesionador social - entre otras cosas porque permite ver al otro como parecido a nosotros y eventualmente permite predecir que tendrá conductas semejantes a las que tendríamos nosotros. Churchland infiere entonces que:

El componente neural relacionado que sospecho que refrenda esta dinámica es el siguiente: la imitación por parte de los muy jóvenes es una señal temprana de una corteza frontal que se desarrolla con normalidad, algo necesario en todos los mamíferos, especialmente los altamente sociales. La chimpancé madre o Rhesus o humana no tiene por qué ser consciente de esa señal. Sólo tiene que responder a la imitación como un rasgo atractivo de sus pequeños [...] La actuación imitativa predice que el bebé tiene los recursos neuronales para aprender lo que necesita para sobrevivir, especialmente en el mundo social, pero también en otros ámbitos. Más concretamente, un bebé que pueda imitar tendrá un cerebro social normal. Si el resto de los elementos son normales, una capacidad de aprendizaje social normal es un buen indicativo de que el niño prosperará, y por tanto merece la pena invertir en ello -hablando en términos biológicos (Churchland, 2012: 175).

6 Hablo aquí de emociones sociales entendiendo por tales aquellas que nos llevan a establecer lazos sociales y no en el sentido de que tengan su origen en el plano social. 
Evidentemente se trata de un modelo en el cual la clave está dada por cierta pretensión naturalizadora. Carece por tanto de sentido, según Churchland, hablar de normatividad en cualquier otro sentido que no sea este de normas prudenciales. Todo imperativo categórico no sería más que un imperativo hipotético que la naturaleza ha disfrazado, con mucha astucia.

Sin embargo, creo que esta perspectiva corre el riesgo de llevar a una suerte de quietismo político. ¿Cómo podríamos en este modelo compatibilizar ciertos ideales como por ejemplo la necesidad de lograr la igualdad de géneros con la naturalización de las formas que adquiere la sociabilidad en nuestro tiempo? Si resulta biológicamente exitoso -y de hecho así ha sido para la especie humana a lo largo de milenios- que haya división sexual del trabajo, cosa que Churchland puede explicar con el hecho de que biológicamente las mujeres están mejor preparadas que los hombres para cuidar a la cría dado que todo su sistema hormonal la dispone a ello, ¿por qué tal forma de organización social tendría que cambiar?

Pero hay una cuestión que me parece aún más problemática que esta tendencia implícita al sostenimiento del status quo, considero que es una cuestión que surge en el planteo metodológico de este «modelo de la naturalización» y que se encuentra ligada con el hecho de que se toma al dato científico como puro, plenamente objetivo y neutral, ${ }^{7}$ incontaminado de teoría. En este modelo, entonces, la evidencia empírica así entendida determina de modo completo la teoría ética, que toma la forma de una etología, más que el de una ética. En estas circunstancias, la neuroética pierde el estatuto de disciplina filosófica, con lo cual no habría aquí tampoco interdisciplinariedad.

\section{4. ¿QUÉ FORMA DE INTERDISCIPLINARIEDAD ADMITE UNA DISCIPLINA FILOSÓFICA?}

Si la neuroética desde su mismo origen se constituye como interdisciplinaria o multidisciplinaria (Farah, 2010; Evers 2010; Racine, 2010), y la ética como disciplina filosófica es parte de las especialidades de las que aquella se nutre, se vuelve necesario pensar cuál es el modo en que una disciplina filosófica puede aportar fructíferamente en su interrelación con

7 La cuestión de los sesgos sexistas en las investigaciones de neurociencia social ha sido largamente discutida por la neuroética feminista en los últimos años, mostrando justamente la importancia de analizar los conceptos con los que la ciencia trabaja en la medida en que estos reproducen sesgos y valoraciones implícitas que impactan en los resultados de estas investigaciones (Fine, 2010; Salles \& Evers, 2014; Reverter, 2016). 
otras disciplinas no filosóficas, sin perder su carácter específico y sin ser subsumida en otros campos del saber. Pues si esto pasara no sería la neuroética un campo de discusión genuinamente interdisciplinario y, por tanto, no podría nutrirse de los saberes que son propios de cada una de las ramas que en ella se encuentran.

Para desarrollar esto con mayor detalle comencemos por dar una descripción más o menos amplia de lo que podemos entender por disciplina filosófica. Evidentemente definir de modo preciso en qué consiste la tarea filosófica es algo notoriamente difícil y controvertido y que excede, por lo tanto, en mucho las pretensiones de este trabajo. Sin embargo, podemos tomar algunas consideraciones que han sido desarrolladas en el ámbito de la metafilosofía para pensar los modos en que la filosofía se relaciona con otras disciplinas, y en especial con las diversas disciplinas científicas. Sven Ove Hansson define a la filosofía por el tipo de metodología específica que emplea, la cual incluye «el uso sistemático de contraejemplos, definiciones precisas y análisis conceptual y análisis lógico de la validez de los argumentos» (Hansson 2008: 476). ${ }^{8}$ Podemos agregar a esto que en toda disciplina filosófica siempre queda abierta la posibilidad de sostener distintas tesis en relación con la cuestión de la que se ocupa la disciplina. La discusión filosófica en sí misma presupone esto en la medida en que lo que se pide es que se presenten argumentos racionales que sustenten la posición adoptada, los que eventualmente pueden incluir datos empíricos, con lo que de todos modos no se salda la cuestión de manera concluyente. David Lewis lo planteaba, hace algunas décadas, en estos términos:

Las teorías filosóficas nunca son refutadas de modo concluyente ( $\mathrm{O}$ casi nunca. Gödel y Gettier pueden haberlo hecho.) La teoría sobrevive a sus refutaciones -a un precio.Argle [personaje del diálogo Holes] ha dicho qué es lo que hacemos en la argumentación filosófica: calculamos el precio. Tal vez eso es algo que podemos resolver de manera más o menos concluyente. Pero cuando todo está dicho y hecho, y todos los argumentos complicados, las distinciones y los contraejemplos se han descubierto, presumiblemente todavía tendremos que hacer frente a la pregunta de qué precio vale la pena pagar, cuáles teorías están en un equilibrio creíble, cuáles son las consecuencias inaceptablemente contraintuitivas y cuáles son las aceptablemente contraintuitivas (Lewis, 1983: x).

8 En cuanto a la potencialidad de la filosofía para articularse con otras disciplinas, Hansson considera que "las herramientas y metodologías desarrolladas en filosofía son útiles tanto en otras disciplinas académicas como en la sociedad en general. Los filósofos pueden contribuir en discusiones complejas, por ejemplo, clarificando el sentido de los conceptos centrales, separando las cuestiones normativas de las empíricas, y distinguiendo entre diferencias lingüísticas y sustanciales en las posiciones en disputa" (481). 
Ciertamente no hay palabra final o argumentación concluyente en ninguna disciplina filosófica. Prácticamente no podemos encontrar ninguna tesis filosófica que no pueda ser sostenida hoy día, aunque sí es cierto que hay períodos de "hibernación» de ciertas posiciones con las matizaciones y puestas al día necesarias. Por ejemplo, luego de que la ética de la virtud parecía no tener mucho para decir frente a la ética deontológica o a los planteos utilitaristas, en el siglo xx, A. McIntyre (1981) o E. Anscombe (1958) han reformulado aquella posición como una respuesta válida ante la pregunta por el bien moral. Podemos estar o no de acuerdo con esta postura, pero sin duda tiene un lugar en la discusión ética y bioética contemporánea. Una ontología monista ${ }^{9}$ o la reintroducción de los poderes causales como elementos básicos en la ontología, y el intento de explicación de las causas en términos de que tales poderes podrían ser otros ejemplos que permiten ver que, en una disciplina filosófica, en este caso en la metafísica, siempre es posible volver a sostener posiciones que en cierto momento se consideraron perimidas (Molnar, 2002; Mumford \& Anjum, 2011).

A lo largo de la historia pueden aparecer tesis nuevas respecto de la problemática propia de una disciplina, por ejemplo, en la ética, en la filosofía política o en la metafísica. En muchos casos estos cambios suelen ir ligados a la aparición de desarrollos científicos que nutren a la filosofía de evidencias novedosas frente a las cuales se hace necesario repensar cuestiones y marcos conceptuales que históricamente han sido pensadas a partir de la experiencia de sentido común. ${ }^{10}$ Puede incluso suceder -o al menos se puede argumentar en favor de que tal cosa ha sucedido- que un determinado problema filosófico mute o incluso se desvanezca por un tiempo. Pero lo que parece no ser aceptable es que una disciplina filosófica nos obligue a adoptar, por la naturaleza misma de la disciplina, como punto de partida una tesis robusta respecto del problema del que se ocupa. Mi punto es que el modelo de interdisciplinariedad al que he nombrado como modelo de la naturalización nos presenta a la neuroética justamente como una disciplina en la cual, para empezar, tenemos que aceptar un punto muy fuerte a nivel teórico: la moralidad es reductible a sus bases neurales. Pareciera que entendida de este modo la neuroética supone la aceptación de que los datos o evidencias aportados por la neurociencia

9 Estrictamente lo que se llama 'monismo de prioridad' —es decir, la tesis de que hay una única cosa fundamental — es una tesis que tiene actualmente seguidores (Schaffer, 2010).

10 Por ejemplo, la filosofía del espacio y el tiempo fue revolucionada por la teoría de la relatividad, o la filosofía de la percepción sensible por los desarrollos en psicología y fisiología. 
explicarán de manera completa el fenómeno moral, pasando incluso por alto que nunca hay dato puro, evidencia sin teoría. Tal vez esta exigencia de la neuroética así entendida explique, en parte, la desconfianza con la que muchos filósofos la miran, pues parece exigirnos asumir desde un principio una posición naturalista y reduccionista, para luego cerrar la discusión.

Para que la neuroética tenga estatuto filosófico es necesario que quede algo por discutir una vez que demos con las bases neurales de la moralidad, e incluso algo por discutir antes de dar con ellas. Pero para hacer esto es necesario que la empresa misma comience de otro modo. No se puede tratar simplemente de la búsqueda de datos empíricos que expliquen conductas humanas. Volviendo a tomar a Churchland como ejemplo, podemos ver que lo que ella hace es tomar la estructura teórica de la biología, con términos teóricos tales como «cambios evolutivos», «selección natural», «genes», etc., y a partir de ese marco interpreta los datos aportados por la neurociencia que le permitirían dar cuenta de la moralidad. Pero ¿si se trata de dar cuenta de la moralidad, los datos de nivel empírico no deberían amoldarse según la estructura teórica de la ética?

Como vemos, la relación entre la evidencia neurocientífica y la reflexión filosófica plantea una serie de cuestiones que merecen atención, como la de si es posible la integración de las metodologías científica y filosófica, y de qué modo o cuál de estas disciplinas tiene mayor autoridad y prioridad epistemológica en la discusión de las cuestiones (Farisco, Evers \& Salles, 2016).

Para dar respuesta, aunque más no sea parcial y preliminar, a algunas de estas cuestiones podemos resumir nuestra posición en relación con el tipo de contribución de la ética en el desarrollo de la neuroética como sigue: muy esquemáticamente podemos pensar que tanto la psicología cognitiva como la neurociencia cognitiva y la neurología aportan datos empíricos, evidencia empírica, que tiene que funcionar como «materia prima» para la tarea teórica del filósofo. Este ha de prestar atención a tal información, evaluará su relevancia y usará de ella para argumentar en favor de una cierta construcción teórica, o incluso encontrará argumentos por los cuáles el dato empírico puede no ser relevante en ciertos casos, de acuerdo con una determinada teoría ética o con determinadas conceptualizaciones metaéticas. ${ }^{11}$ Es decir, la evidencia ha de volver necesario para el filósofo

11 La "neuroética conceptual" propuesta por A. Salles y K. Evers (en prensa) y la "neuroética pragmática" que plantea J. Shook y J. Giordano (2016), con sus particularidades, podrían tomarse como ejemplos de este tipo de modelo interdisciplinario. 
argumentar tanto por qué y de qué modo el dato ocupa un lugar en la teoría como por qué no tiene relevancia, en caso de que esto fuera así.

Podríamos intentar dar una lista no exhaustiva de algunas de las tareas que suponen una perspectiva filosófica en el interior de la neuroética.

En primer lugar, aclarar y definir de modo preciso los conceptos a partir de los cuales se diseñan las investigaciones neurocientíficas, para poder determinar las implicancias de los resultados que se siguen de tales investigaciones. Un ejemplo en el que se pueden notar los malentendidos a los que da lugar la falta de análisis filosófico han sido las conclusiones a las que arriba Benjamin Libet a partir de sus experimentos referidos a la temporalidad de la toma de conciencia ante una elección, y a los que pone en relación con el libre arbitrio (Libet 1999). Si bien posteriores experimentos neurocientíficos han invalidado las conclusiones de Libet, ${ }^{12}$ puede argumentarse que tales experimentos no ponían, en verdad, en cuestión la libertad de la voluntad sino una concepción particular de esta, aquella que identifica libertad con indeterminación, posición que, por otra parte, tiene muy pocos seguidores en el ámbito filosófico (Bouger \& Chalmers, 2014). Un análisis conceptual adecuado, así como el conocimiento del desarrollo histórico de tal concepto, permitirían investigaciones neurocientíficas más fructíferas. $^{13}$

En segundo lugar, interpretar la evidencia aportada por la investigación en neurociencia cognitiva tendiente a explicar el comportamiento moral para determinar de qué modo tales evidencias se compatibilizan o armonizan con las distintas teorías éticas. Vuelvo aquí a recordar la observación de David Lewis, citada más arriba, respecto de la imposibilidad de agotar plenamente la discusión entre teorías filosóficas, incluso a partir de datos empíricos.

En tercer lugar, identificar y deslindar el carácter descriptivo del carácter prescriptivo presente en las investigaciones neurocientíficas es también una tarea filosófica propia de la neuroética. Tal como señalan J. Shook y J. Giordano, «el modo en que la neurociencia de la ética recomienda ajustes en nuestras concepciones del yo, la moralidad y la sociedad necesariamente involucra importantes cuestiones normativas y éticas» (2014: 2). Una neuroética que pretenda aportar una mera descripción del «modo en

12 Una revisión bibliográfica al respecto se presentó en el IV Congreso Internacional de Bioética de Valencia (Costa Alcaraz et al., 2016).

13 El tipo de trabajo que hace Neil Levy (2011 y 2012) podría ejemplificar este tipo de análisis que permite aclarar y precisar los conceptos éticos y metaéticos implícitos en las investigaciones neurocientíficas. 
que los humanos hacen las cosas» encubre su carácter prescriptivo bajo una fachada de convencionalidad cultural no reconocida como tal.

\section{CONCLUSIÓN}

La neuroética presenta en la actualidad un interesante desafío para pensar de qué modo es posible la interdisciplinariedad en un campo en el que se cruzan la ciencia empírica y la filosofía.A lo largo de este trabajo hemos tratado de distinguir dos modelos metodológicos en que se ha plasmado esta relación disciplinar en los trabajos de importantes neuroeticistas y señalamos las dificultades y tensiones que se plantean en esos dos modelos para articular los aportes que la ética y la neurociencia social pueden hacer para una mejor compresión de la moralidad y de los conceptos éticos y metaéticos.

En relación con el primer modelo, el de la neuroética filosófica, señalamos que más que un conocimiento surgido de una genuina interdisciplinariedad consiste más bien en tomar aquellos datos que son relevantes para fundamentar una teoría ética particular a la que se considera válida con independencia de tal evidencia.

En cuanto al modelo de la naturalización, sostenemos que no da lugar a una relación fructífera entre disciplinas porque asume desde el comienzo que el dato científico agota la discusión acerca de la cuestión ética, e identifica además el plano descriptivo con el normativo.

Finalmente, presentamos algunos lineamientos generales para pensar en qué puede consistir la tarea de reflexión ético-filosófica en el interior de la neuroética, pensada esta como un campo genuinamente interdisciplinar.

\section{AGRADECIMIENTOS}

Agradezco a Arleen Salles y a Ezequiel Zerbudis por los comentarios y sugerencias que hicieron a versiones previas de este trabajo.

\section{BIBLIOGRAFÍA}

Álvarez-Díaz, Jorge Alberto (2013). Neuroética como neurociencia de la ética. Revista de Neurología, 57, 374-82. 
Anscombe, Gertrude E. M. (1958). Modern Moral Philosophy. Philosophy, 33(124), 1-19.

Berker, Selim (2009). The Normative Insignificance of Neuroscience. Philosopby \& Public Affairs, 37, 293-329.

Bourget, David \& Chalmers, David (2014). What do philosophers believe? Philosophical Studies, 170(3):465-500.

Buller,Tom (2006). What can neuroscience contribute to ethics? Journal of Medical Etbics, 32, 63-4.

Churchland, Patricia (2012). El cerebro moral. Barcelona: Paidós.

Conill Sancho, Jesús (2017). ¿Tiene arraigo en el cerebro la libertad? Pensamiento: Revista de Investigación e Información Filosófica, 73(276), 493-514.

Cortina, Adela (2011). Neuroética y neuropolítica. Sugerencias para la educación moral. Madrid:Tecnos.

Cortina, Adela (2013). Ética del discurso: ¿un marco filosófico para la neuroética? Isegoría, 48, 127-48.

Cortina, Adela (2014). Neuroética y ética del discurso. En Salles, Arleen \& Evers, Kathinka, La vida social del cerebro. México DF: Fontamara.

Costa-Alcaraz,Ana María, Ruiz-Ripoll,Ada I. \& Vázquez-Costa, Juan Francisco (2016). Aportaciones de las neurociencias al razonamiento moral. IV Congreso Internacional de Bioética: Bioética y Razonamiento Moral.Valencia.

Evers, Kathinka (2010). Neuroética: Cuando la materia se despierta. Buenos Aires: Katz.

Evers, Kathinka, Salles, Arleen \& Farisco, Michele (2017). Theoretical Framing of Neuroethics: The Need for a Conceptual Approach. En Racine, Erik \& Aspler, John, Debates About Neuroetbics. Advances in Neuroetbics (89-107). Cham: Springer.

Farah, Martha (2010). Neuroetbics: An Introduction with Readings. Cambridge: MIT Press.

Farisco, Michele, Evers, Kathinka \& Salles, Arleen (2016). Big Science, Brain Simulation, and Neuroethics. AJOB Neuroscience, 7(1), 28-30.

Fine, Cordelia (2010). Delusions of Gender: How Our Minds, Society, and Neurosexism Create Difference. Nueva York: W. W. Norton.

Gazzaniga, Michel (2005). The Ethical Brain. Nueva York: Dana.

Giordano, James (2011). Neuroethics:Traditions, tasks and values. Human Prospect, 1(1): 2-8.

Greene, Joshua, \& Haidt, Jonathan (2002). How and where does moral judgment work? Trends in Cognitive Science, 6(12), 517-523. 
Greene, Joshua (2008). The Secret Joke of Kant's Soul. En Sinnott-Armstrong, Walter, Moral Psychology, vol. 3: The Neuroscience of Morality (35-79). Cambridge: MIT Press.

Greene, Joshua (2014). Beyond point-and-shoot morality: Why cognitive (neuro)science matters for ethics. Ethics, 124(4), 695-726.

Haidt, John (2001). The emotional dog and its rational tail, a social intuitionist approach to moral judgment, Psychological Review, 108(4), 814834.

Hansson, Sven Ove (2008). Philosophy and other disciplines, Metapbilosopby, 39(4-5), 472-483.

Illes Judy, Kirschen, Matthew P. \& Gabrieli, John D. (2003). From neuroimaging to neuroethics. Nature Neuroscience. 6(3), 205.

Joyce, Richard (2008). What neuroscience can (and cannot) contribute to metaethics. En Sinnott-Armstrong, Walter (ed.), Moral Psychology, volume 3: The Neuroscience of Morality: Emotion, Brain Disorders, and Development (371-394). Cambridge: MIT Press.

Levy, Neil (2012). Neuroethics. WIREs Cognitive Science, 3(2), 143-151.

Levy, Neil (2011). Neuroethics: a new way of doing ethics. AJOB Neuroscience, 2, 3-9.

Lewis, David (1983). Philosophical Papers, vol. 1, Oxford: Oxford University Press.

Libet, Benjamin (1999). Do we have free will. Journal of Consciousness Studies 6, 47-57.

MacIntyre, Alasdair (1981). After virtue: $A$ study in moral theory. Notre Dame: University of Notre Dame Press.

Marcos, Adolfo (2015). Neuroética y vulnerabilidad humana en perspectiva filosófica. Cuadernos de Bioética, 26(3), 397-414.

Marcus, Steven (ed.) (2004). Neuroethics: Mapping the Field; Conference Proceedings, May 13 - 14, 2002, San Francisco, California. Nueva York: Dana.

Molnar, George (2003). Powers. Oxford: Oxford University Press.

Mumford, Stephen \& Anjum, Rani (2011). Getting Causes from Powers. Oxford: Oxford University Press.

Northoff, Georg (2009). What is neuroethics? Empirical and theoretical neuroethics. Current Opinion in Psychiatry, 22(6): 565-569.

Parens, Eric \& Johnston, Josephine (2007). Does it make sense to speak of neuroethics?: Three problems with keying ethics to hot new science and technology. EMBO Reports, 8(supl 1), 61-64. 
Racine, Eric (2010). Pragmatic Neuroethics: Improving Treatment and Understanding of the Mind-brain. Cambridge: MIT Press.

Reverter Bañón, Sonia (2016). Reflexión crítica frente al neurosexismo. Pensamiento. Revista de Investigación e Información Filosófica, 72(273), 959-979.

Roskies, Adina (2002). Neuroethics for the New Millenium. Neuron, 35(1), 21-23.

Roskies, Adina (2016). Neuroethics. The Stanford Encyclopedia of Philosophy [versión en línea] Standford, CA: The Metaphisics Research Lab. Retrived from:https://plato.stanford.edu/archives/spr2016/entries/neuroethics/.

Safire, William (2004). Introduction. En Marcus, Steven (ed.). Neuroethics: Mapping the Field; Conference Proceedings, May 13 - 14, 2002, San Francisco, California. Nueva York: Dana.

Salles, Arleen \& Evers, Kathinka (eds.) (2014). La vida social del cerebro, México: Fontamara.

Salles, Arleen \& Evers, Kathinka (en prensa). Social neuroscience and Neuroethics: A Fruitful Synergy. En Ibáñez, A., Sedeno, Lucas, \& Garcia, Adolfo (eds.). Social Neuroscience and Social Science: The Missing Link. Nueva York: Springer.

Schaffer, Jonathan (2010). Monism:The Priority of the Whole. Philosophical Review, 119, 31-76.

Shook, John \& Giordano, James (2014). A Principled and Cosmopolitan Neuroethics: Considerations for International Relevance. Philosophy, Ethics, and Humanities in Medicine, 9(1), 2-13. 